\title{
Ultrasound Training for Obstetrics and Gynecology Residents
}

Carla A Martinez ${ }^{1}$, Anjana R Nair ${ }^{2}$, Sanja Kupesic-Plavsic ${ }^{3}$

\begin{abstract}
The purpose of ultrasound training for obstetrics and gynecology (OB-GYN) residents is to advance the safe and effective use of transabdominal and transvaginal sonography in reproductive medicine, infertility, gynecologic oncology, urogynecology, normal, and abnormal pregnancy. The purpose of obstetrical ultrasound training is to provide an integrated approach of hands-on ultrasound training, required readings, and web-based lecture series around obstetrics. The creation of the pelvic ultrasound curriculum was a part of a research project, which resulted in multiple national and international presentations and publications.

Every year of their postgraduate training, the residents spend 1 month in the ultrasound clinic, working through various cases to build technical skills in performing OB-GYN ultrasounds. The training begins with a review of the institution-specific online modules, followed by weekly rotation/hands-on training sessions and periodic ultrasound simulations. Throughout the 4 years of training the resident spends designated time in the ultrasound clinic as well as gain hands-on training on inpatient antepartum patients.

Comprehensive training allows residents to perform a well-structured ultrasound examination, write a structured report and graduate with competency in OB-GYN ultrasound. It is designed to help them develop the fundamental knowledge, theoretical background, and mastery of skills of effective diagnostic ultrasound that can be incorporated in their clinical practice postresidency.

Keywords: OB-GYN residency, OB-GYN ultrasound curriculum, OB-GYN ultrasound training, Obstetric ultrasound, Pelvic ultrasound. Donald School Journal of Ultrasound in Obstetrics and Gynecology (2021): 10.5005/jp-journals-10009-1801
\end{abstract}

\section{INTRODUCTION}

Diagnostic ultrasound imaging is the most used imaging modality in obstetrics and gynecology (OB-GYN). In the United States and Europe, ultrasound has become an established part of the curriculum in OB-GYN residency programs. Residents are expected to be proficient in understanding, performance, and interpretation of pelvic ultrasound examinations. ${ }^{1,2}$ American Institute for Ultrasound in Medicine (AIUM) published an ultrasound curriculum for OB-GYN postgraduate training programs in the United States. The program provides a comprehensive introduction to ultrasound, as well as focused training in OB-GYN imaging. On-site faculty instructors are encouraged to use this curriculum for standardized ultrasound education across the United States. ${ }^{3}$

Here we discuss our experience with creating and implementing the institution-specific pelvic and obstetric ultrasound curriculum for OB-GYN residents.

\section{Pelvic Ultrasound Curriculum}

In 2015, Green et al. evaluated the state of ultrasound education among Canada's 16 postgraduate training programs in OB-GYN ultrasound. ${ }^{4}$ Their results confirmed a suspected gap in resident exposure to GYN-specific ultrasound training. While most residency programs in Canada provided a formal OB ultrasound curriculum consisting of didactics and hands-on, a smaller percentage of programs reported having a formal GYN ultrasound curriculum and hands-on experience. Two-thirds of OB-GYN residents in Canada thought that their current training in $\mathrm{OB}$ ultrasound would adequately prepare them for practice, while less than one-third believed that they would be sufficiently trained in GYN ultrasound. ${ }^{4}$

The pelvic ultrasound curriculum for OB-GYN residents in our institution was developed to ensure an adequate understanding of

\footnotetext{
${ }^{1-3}$ Department of Obstetrics and Gynecology, Texas Tech University Health Sciences Center El Paso, El Paso, Texas, USA

Corresponding Author: Sanja Kupesic-Plavsic, Department of Obstetrics and Gynecology, Texas Tech University Health Sciences Center El Paso, MSC 21007, 5001 El Paso Drive, El Paso, Texas, USA, Phone: +1 9152155065, e-mail: sanja.kupesic@ttuhsc.edu

How to cite this article: Martinez CA, Nair AR, Kupesic-Plavsic S. Ultrasound Training for Obstetrics and Gynecology Residents. Donald School J Ultrasound Obstet Gynecol 2021;15(4):352-358.

Source of support: Nil

Conflict of interest: None
}

ultrasound evaluation of the most common gynecologic disorders. The curriculum consists of the online modules in TTUHSC El Paso Learning Management System (LMS), Canvas, hands-on training sessions, and periodic ultrasound simulation sessions. The purpose of the Pelvic Ultrasound hybrid curriculum was to advance the safe and effective use of ultrasound examination in reproductive medicine, infertility, gynecologic oncology, urogynecology, and assessment of normal and abnormal early pregnancy.

The curriculum was created by an OB-GYN professor (SKP) with more than thirty years of experience in academic medicine, reproductive endocrinology, OB-GYN ultrasound, and ultrasound-guided procedures.

The trainees are provided with the pelvic ultrasound course syllabus to familiarize themselves with the course content, learning objectives, course organization, technical requirements, and expectations. For interns and/or first-year residents, this may be their first experience with canvas. To familiarize themselves with the LMS, they are provided with the navigation guide and online

(OTheAuthor(s). 2021 Open Access This article is distributed under the terms of the Creative Commons Attribution 4.0 International License (https://creativecommons. org/licenses/by-nc/4.0/), which permits unrestricted use, distribution, and non-commercial reproduction in any medium, provided you give appropriate credit to the original author(s) and the source, provide a link to the Creative Commons license, and indicate if changes were made. The Creative Commons Public Domain Dedication waiver (http://creativecommons.org/publicdomain/zero/1.0/) applies to the data made available in this article, unless otherwise stated. 
instruction, which takes them step-by-step through the basics of navigating the canvas.

The online modules are structured to complement hands-on experience during pelvic ultrasound rotation and could be accessed and completed anytime, anywhere, and at their own pace. Each module consists of a brief module description, learning objectives, five pretest questions, educational video, suggested readings, and summative examination consisting of 10 post-test questions (Fig. 1). An online ultrasound course is organized around major clinical topics with relevant objectives, easily "digestible" high-resolution videos with narrated explanations. The narrated PowerPoints (PPTs) are short (between 10 and 20 minutes), which maximizes residents' attention, enables them to familiarize themselves with interpretation and ultrasound vocabulary, absorb the information, and avoid overload.

Multiple choice quizzes at the end of each module assist the learners to recall information and apply the knowledge. To promote mastery of the material, learners are allowed to take each quiz three times, with no time allocation for each attempt. Multiple choice questions with a maximum of five possible answers (from "a-e") objectively assess learner knowledge after going over the educational material. An $80 \%$ score must be achieved in the post-test quiz to receive credit. After completing the quiz, residents are invited to join a discussion forum by asking questions, sharing thoughts, and providing suggestions for improvement of the online content and clinical rotation experience.

The lead analysts in the office of faculty development assist in placing the online quizzes onto canvas and select appropriate settings to allow for automatic grading of the OB-GYN residents. The highest grade obtained on any attempt is recorded, and a quarterly report is sent to the Residency Program Director.

The pelvic ultrasound online course consists of the seven modules, organized according to the Paul L. Foster School of Medicine clinical presentation-based curriculum:

- Introduction

- How to conduct a pelvic ultrasound examination?

- How to write a pelvic ultrasound report?

- Normal pelvic anatomy

- Female infertility

- Uterine lesions
- Adnexal lesions

- Pelvic pain

- Abnormal uterine bleeding.

The first module focuses on normal pelvic anatomy, gynecologic and obstetrical indications for pelvic ultrasound (Tables 1 and 2), and equipment specifications. Transabdominal and transvaginal scanning techniques, transducer preparation, orientation, and manipulation are explained in detail. The module discusses the sonographic techniques used for pelvic evaluation. The size, shape, and positional variants of the uterus and the ovaries relative to the age and the phase of the menstrual cycle are explained in detail. Sonographic appearance and the relationship between the gynecologic, bowel, and urinary tract structures are described and explained. The module summarizes transabdominal sonography (TAS) and transvaginal sonography (TVS) examination techniques, use of color, power, and spectral Doppler, as well as the best practices of three-dimensional (3D) ultrasound imaging of the pelvic organs.

The second module is dedicated to ultrasound assessment of the menstrual cycle by the two-dimensional (2D), spectral Doppler, and 3D ultrasound.

In the third module, the learners are familiarized with the ultrasound findings of the ovarian, tubal, and uterine causes of infertility.

The modules on uterine and adnexal masses illustrate ultrasound findings of the most common benign and malignant uterine and adnexal masses.

The pelvic pain module discusses ultrasound findings of the patients presenting with acute and chronic pelvic pain.

Finally, the module on abnormal uterine bleeding presents the most common ultrasound findings of the patients presenting with reproductive-age and postmenopausal uterine bleeding.

Residents appreciate Pelvic Ultrasound Online Course flexibility and its instant relevance to the pathology they see in the clinic and on pelvic ultrasound rotation. Accessibility of the course material 24/7 allows them to study around their work schedule. Consistency of recorded and uploaded learning material, incorporation of multiple media, objective knowledge assessment, and immediate feedback are the most important benefits of this hybrid instruction.

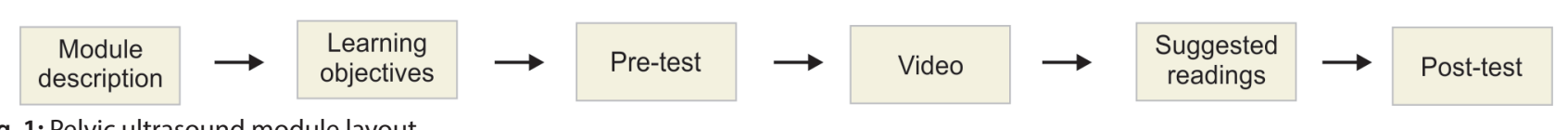

Fig. 1: Pelvic ultrasound module layout

Table 1: List of gynecologic indications for pelvic ultrasound encounters

Detection and evaluation of uterine masses

Abnormal uterine bleeding in adolescent, reproductive age, and peri- and postmenopausal patients

Detection of uterine malformations

Monitoring of the follicular growth and endometrial changes

Periovulatory monitoring of endometrial, cervical, and circulatory changes

Detection and evaluation of adnexal masses

Evaluation of posterior cul-de-sac

Evaluation of nongynecologic structures

Table 2: List of obstetrical indications for pelvic ultrasound encounters

\begin{tabular}{l} 
Obstetrical indications for transvaginal sonography \\
Early detection of intrauterine pregnancy \\
Early dating by biometry \\
Evaluation of multiple pregnancy (location, numeration, \\
chorionicity, and amnionicity) \\
Detection and evaluation of early pregnancy failure and pathology \\
Evaluation of patients with direct and indirect signs of ectopic \\
pregnancy \\
Detection of embryonic and early fetal anomalies \\
Diagnosis of placenta previa \\
Cervical incompetency \\
Evaluation of intracranial structures in cephalic presentations \\
\hline
\end{tabular}

Urogynecologic evaluation

Evaluation of intracranial structures in cephalic presentations 
The goals of pelvic ultrasound rotation are to understand the basic principles of pelvic ultrasound and integrate them into patients' diagnostic workup and management. Indications for a pelvic ultrasound, "knobology," image acquisition, and interpretation are thoroughly discussed. Free access to online ultrasound reference books, journal articles, multimedia resources, online modules, and question banks enrich residents' exposure to online active learning. Interaction with sonographers and scanning gynecologic patients under supervision contributes to their interprofessional, collaborative, and interactive educational experience which encourages them to scan their patients throughout the year.

\section{Pelvic Ultrasound Rotation}

In many U.S. academic institutions, radiology departments are responsible for performing pelvic ultrasounds. Due to the small volume of GYN ultrasound studies and lack of qualified supervision, many OB-GYN residents feel less confident performing and interpreting pelvic than $\mathrm{OB}$ ultrasound examinations.

Our ultrasound curriculum was created according to the AIUM recommendations and guidelines. ${ }^{3}$ The author of the online course/modules and onsite faculty trainer is knowledgeable of the milestones for ultrasound scanning for each OB-GYN residency year. During rotation in the ultrasound clinic, residents work through various cases to build their technical skills and knowledge in performing and interpreting pelvic ultrasounds. Residents discuss the clinical information and the format of the written and/or electronic request for pelvic ultrasound examination with their supervisor. After conducting transabdominal and transvaginal scans, they are provided with immediate feedback.

Throughout their rotation, residents are instructed how to perform a systematic pelvic ultrasound examination, objectively describe and interpret sonographic findings, and write a complete ultrasound report. They are expected to compare the investigations that were or have to be performed for each indication and present the information on previous ultrasound findings. The trainees are also introduced to sonohysterography or saline infusion sonography (SIS) in reproductive medicine.

\section{Pelvic Ultrasound Simulation}

Our OB-GYN residents have the benefit of using a high-end simulation center (Training and Educational Center for Healthcare Simulation, TECHS at TTUHSC EI Paso), where they utilize pelvic ultrasound simulation in a safe, effective, and learner-centered educational environment. Currently, our simulation center has three types of ultrasound simulators:

- Online: Mouse-operated web-based programs that control and change scan planes and simulate probe manipulation. The display demonstrates ultrasound images corresponding to the particular scan plane.

- High-fidelity mannequins: Consist of the mannequin, simulator, ultrasound probe, computer, and monitor. The display uses virtual anatomic model images with augmented reality and ultrasound-rendered images or actual ultrasound images from a stored dataset.

- Phantoms: Use of a real ultrasound unit to image a phantom to practice diagnostic and/or procedural skills (e.g., amniocentesis phantom).
Patient encounters with novices who do not have appropriate training may compromise patient care by request for unnecessary intervention, and additional testing. ${ }^{5,6}$ In a survey of 70 OB-GYN residents, $50 \%$ of them reported a failure to achieve ultrasound competencies required for their stage of training. Seventy-three percent (73\%) considered ultrasound simulation to be an essential component of their residency training which may improve their clinical and interpretation skills. ${ }^{7}$

Madsen et al. ${ }^{8}$ reported that the virtual reality (VR) simulator is a reliable and valid tool to improve pelvic ultrasound examination skills. ${ }^{8}$ Their study recorded an improvement in novices' performance, which plateaued after 4 hours of simulation training. Girzadas et al. ${ }^{9}$ used a hybrid pelvic ultrasound simulation consisting of a transvaginal ultrasound task trainer combined with a high-fidelity ultrasound mannequin. ${ }^{9}$ Residents enrolled in this type of simulation demonstrated improved knowledge, diagnostic, and management skills in the diagnosis and treatment of a ruptured ectopic pregnancy case. ${ }^{9}$ Similarly, Vallabh-Patel et al. ${ }^{7}$ and Monsky et al. ${ }^{10}$ reported improvement in clinical knowledge, scanning ability, and interpretation skills in a clinical setting after using low- and high-fidelity transabdominal and transvaginal pelvic ultrasound simulators. Furthermore, pre- and postsimulation learner assessment revealed improved image optimization and probe orientation. ${ }^{7}$ A systematic review by Arya et al. ${ }^{11}$ assessing the use of ultrasound simulation in OB-GYN training reported a significant improvement in clinical knowledge, skills, and behaviors of the trainees.

Patient discomfort and the intimate nature of transvaginal sonography encourage the need for simulation-based training. The opportunity for unlimited practice without pressure facilitates a systematic approach to pelvic ultrasound scanning. ${ }^{12}$

A recent study by Alhramani et al. ${ }^{13}$ assessed ultrasound-training curricula in Accreditation Council for Graduate Medical Education (ACGME) approved OB-GYN residency programs across the United States. They obtained responses from 93 program directors and 437 residents. Eleven percent of program directors and $23 \%$ of residents reported a lack of ultrasound-related didactics. Of the residency programs with didactics, $27 \%$ of program directors and $40 \%$ of residents had it yearly or less. Three quarters of programs had mandatory ultrasound rotations. Most respondents stated that the main focus of ultrasound rotation was OB only. ${ }^{13}$ Scanning skill was evaluated mainly subjectively by direct observation. Most 4th-year residents (76\%) stated that they would require additional training to perform or read ultrasound examinations independently, and $43 \%$ would not treat a patient based on their ultrasound examinations without further confirmation. ${ }^{13}$ Subsequent studies confirmed the need for a standardized OB-GYN ultrasound curriculum, with an equal focus on $O B$ and GYN ultrasound. ${ }^{13-15}$

\section{Obstetric Ultrasound Curriculum}

Basic ultrasound knowledge, image interpretation, and skillsets are vital for the practicing obstetrician. Surveys of ultrasound training in OB-GYN residency programs have varied over the decades. ${ }^{13,16,17}$ While access to the modalities has improved since the initial survey in 1987 , up to $20 \%$ of the residency program directors would not base treatment of their private patients based on their senior resident's ultrasound examination..$^{13,16}$

American College of Obstetrics and Gynecologists (ACOG) and the AIUM for years have published guidelines for the performance of obstetric ultrasound; however, before 2018, there was not a recommended curriculum. ${ }^{1,18}$ Prior to this several 
institutions published their experience and OB-GYN curriculums based on guidelines at the time. ${ }^{18,19}$ In 2018, AIUM published a consensus-based, standardized ultrasound curriculum and competency tool for OB-GYN residency programs. ${ }^{3}$ This curriculum could help overcome the lack of clearly defined milestones and heterogeneity observed in OB-GYN ultrasound curricula..$^{13}$ It outlines clearly defined objectives based on the level of training from years 1-4 with recommended competency assessments. ${ }^{3}$ Current education objectives from the Council on Resident Education in Obstetrics and Gynecology (CREOG) for ultrasonography are limited to assessing the fetal number, lie, presentation, viability, placental location, fetal biometry, cervical length, ability to perform, and interpret a biophysical profile (BPP); and describing indications for assessment with Doppler ultrasonography (e.g., umbilical artery and middle cerebral artery).$^{20}$ The recommended AIUM curriculum offers an in-depth training curriculum that allows the trainee to achieve the CREOG objectives and structured training. Here we discuss our experience with creating and implementing a hybridization of the AIUM-recommended curriculum and CREOG objectives.

The OB ultrasound curriculum for OB-GYN residents at our institution was developed to meet the educational objectives of CREOG as well as incorporate the AIUM recommended curriculum. Residents are provided with the ultrasound course syllabus which includes course content, learning objectives, course organization, expectations, and required readings. At our institution, resident training in ultrasound begins during their intern boot camp with an introduction to the official statements by ACOG as well as the AIUM ultrasound curriculum..$^{3,17,18}$ Interns are introduced to the ultrasound unit by spending half a day with a registered diagnostic medical sonographer (RDMS) to begin to gain experience with basic characteristics of the ultrasound equipment and bioeffects of ultrasound. They are supervised by maternal-fetal medicine (MFM) subspecialists. They begin hands-on scanning to be able to begin to assess fetal number, lie, presentation, viability, and the placental location. The goal is to begin to familiarize themselves with the ultrasound machine and basic knobology in preparation for their time spent in labor and delivery. The ultrasound machines used in the clinic and hospital are all from the same manufacturer and models. This has allowed us to be consistent across locations and providers feel more comfortable using the machines as the platforms are the same. To eliminate missing elements of examinations, due to lack of experience, we use the ultrasound scan assistant to assist with each of the basic examination types (limited second or third-trimester obstetric examination, BPP, and cervical length) for examinations done in the labor and delivery triage unit. These examinations are typically supervised by a general obstetrician attending covering the unit.

The residents are provided two resources to aid in image recognition and parameters for obtaining standard obstetrical views into both the first and second trimesters. 3,19,21 The images provided in these references are also used in pre-and post-quiz that are taken at the beginning and end of each 1-month rotation. The residents are given an outline of the month and goals in acquiring images with each week focused on a different organ system. As the residents progress in their learning and ability to acquire images they are then assessed at the end of each day and given oral feedback from the RDMS as well as the MFM. At the end of each week, they are then evaluated with an objective assessment by both the RDMS and the MFM for each of the areas they worked on for the week (Tables 3 to 5). As they progress through aspects of the anatomy scan (Table 1), they are evaluated based on the sections completed during the scanning session, with each section having the minimum number of images needed to pass that section. At the end of their residency training, the goal for them is to be able to complete an entire anatomical survey with fetal biometry and obtain a cervical length.

We also utilize the online OB-GYN ultrasound course lecture series program provided by CREOG. ${ }^{20}$ The project was developed by the AIUM with input from several collaborating organizations that include: ACOG, CREOG, American College of Radiology (ACR), American College of Osteopathic Obstetricians (ACOOG), International Society of Ultrasound in Obstetrics and Gynecology (ISUOG), Society for Maternal-Fetal Medicine (SMFM), and the Society of Radiologists in Ultrasound (SRU). The program content includes a comprehensive in-depth curriculum that goes beyond the scope of the CREOG educational objectives and milestones. We focus on the highest yield core topics for OB-GYN residency training but encourage them to complete the entire series of lectures.

The time spent in OB ultrasound is combined with their MFM rotation. During each 1-month rotation, they are provided a minimum of 3 days of hands-on ultrasound scanning with both an RDMS and back scanning with an MFM. Incorporated into their learning are both normal and abnormal ultrasound imaging and counseling of patients by the MFM. In addition to the AIUM lecture series, to assist with learning abnormal ultrasound imaging we review the SMFM fetal anomalies consult series. ${ }^{22-26}$ These references are utilized to review abnormalities and are incorporated into the curriculum as new topics are published. The consulting series is focused more on the subspecialist MFM and MFM fellows, but it can help prepare the generalist OB-GYN with additional knowledge and as a reference as each anomaly follows a standard template which includes delivery management and prognosis. This allows incorporation of teaching beyond only normal fetal ultrasounds and allows them to gain experience with observing diagnostic procedures as well a multidisciplinary care of the patient and her fetus.

A recent survey that assessed resident comfort with obstetrical ultrasounds demonstrated $81 \%$ felt comfortable independently performing growth ultrasound after residency. Of those that felt comfortable, they came from smaller programs with less than seven residents, had longer MFM rotations, did not plan on doing an MFM fellowship, and did not have the presence of MFM fellows. ${ }^{26}$ Of those that had experience with anatomy ultrasounds, less than a third felt comfortable performing an anatomy ultrasound independently and $50 \%$ less likely to be in programs with MFM fellows present. ${ }^{26}$ While the response rate was low, just over $8 \%$, it was representative of both academic and community training programs and demonstrated a self-reported lack of comfort with obstetrical ultrasound training across the United States. ${ }^{26}$

Integration of obstetrical ultrasound into resident training has shown improved overall comfort with growth ultrasounds. ${ }^{26}$ However, most residents and their supervising attending would not base decisions solely on residents' scan and continued integration of the recommended AIUM curriculum is needed in training programs. ${ }^{3,16,26}$ Integration of the AIUM curriculum into OB-GYN residency will help achieve and surpass required CREOG objectives. 
Table 3: Checklist for second trimester anatomy

Resident name:
Second-trimester anatomy ultrasound
Anatomical structures

Anatomical structures

Fetal presentation (4/5)

Placental position

Placental cord insertion

Amniotic fluid (AFI or DVP)

Transvaginal cervical length

Intracranial anatomy $(5 / 7)$

BPD and HC

CSP, falx, thalami

Lateral ventricles

Choroid plexus

Cerebellum

Cisterna magna

Nuchal fold if applicable

Face $(2 / 3)$

Profile and nasal bone

Nose/lips

Spine $4 / 6$

Cervical spine axial/ midsagittal

Thoracic spine axial/midsagittal

Lumbar/sacral spine axial/midsagittal

Cardiac (3/5)

Heart rate in M-mode and pulsed-wave Doppler

Four chamber

Situs

Intra-abdominal (5/6)

Stomach

Abdominal circumference

Cord insertion

Kidneys

Bladder

Three-vessel cord

Extremities (4/6)

Femur length

Humerus length

Upper extremity, with hand if possible

Lower extremity, with foot if possible

Other extra credit

LVOT

RVOT

Gender

Evaluated by:

$\mathrm{AFI}$, amniotic fluid index; MVP, maximal vertical pocket; BPD, biparietal diameter; $\mathrm{HC}$, head circumference; CSP, cavum septi pellucidi; LVOT, left ventricular outflow tract; RVOT, right ventricular outflow tract
Y

\author{
Structure identified? \\ YES / NO \\ YES / NO \\ Adequate image obtained? \\ Date:

YES / NO YES / NO


Table 4: Checklist for first trimester ultrasound

\begin{tabular}{ll}
\hline $\begin{array}{l}\text { Resident name: } \\
\text { First-trimester ultrasound }\end{array}$ & Date: \\
$\begin{array}{l}\text { Anatomical structures } \\
\text { Adequate im- } \\
\text { age obtained? } \\
\text { YES / NO }\end{array}$ & $\begin{array}{c}\text { Structure } \\
\text { identified? }\end{array}$ \\
Gestational sac & \\
Yolk sac & \\
Fetal pole & \\
Heart rate in M-mode & \\
Uterus measured in two orthogonal \\
dimensions \\
Right ovary measured in two or- \\
thogonal dimensions \\
Left ovary measured in two orthogo- \\
nal dimensions \\
Evaluated by:
\end{tabular}

Table 5: Biophysical profile

\begin{tabular}{|c|c|c|}
\hline Resident name: & \multicolumn{2}{|l|}{ Date: } \\
\hline Biophysical profile & & \\
\hline Anatomical structures & $\begin{array}{l}\text { Adequate im- } \\
\text { age obtained? }\end{array}$ & $\begin{array}{c}\text { Structure } \\
\text { identified? }\end{array}$ \\
\hline & YES / NO & YES / NO \\
\hline
\end{tabular}

Breathing: 30 second periods

Movement: Three 3 gross fetal body or limb movements

Tone: Episode of flexion and extension of fetal $\operatorname{limb}(\mathrm{s})$

Amniotic fluid volume by AFI or MVP

Evaluated by:

Breathing, movement, and tone documented by movie clips if possible; $\mathrm{AFI}$, amniotic fluid index; MVP, maximal vertical pocket

\section{Conclusion}

We describe a hybrid of the AIUM recommended OB-GYN ultrasound curriculum, CREOG objectives, online AIUM lectures, and supervised patient scanning encounters. Incorporating a training curriculum and consistent evaluation enhances the performance and diagnostic accuracy of ultrasound in OB-GYN. The hybrid ultrasound curriculum, consisting of the online modules, supervised patient encounters, and periodic pelvic ultrasound simulation, described in this article, seems to improve residents' clinical knowledge, skills, and competency in performing transabdominal and transvaginal sonography. The best outcomes are achieved when ultrasound rotations are regularly and objectively assessed by qualified staff and supervisors.

\section{References}

1. Hall R, Ogburn T, Rogers RG. Teaching and evaluating ultrasound skill attainment: competency-based resident ultrasound training for AIUM accreditation. Obstet Gynecol Clin North Am 2006; 33:305-323. DOI: 10.1016/j.ogc.2006.02.001

2. Council on Resident Education in Obstetrics and Gynecology. Educational objectives: Core Curriculum in Obstetrics and
Gynecology, 12th ed. Available from: https://www.acog.org/ education-and-events/creog/curriculum-resources/creogeducational-objectives; Accessed May 29, 2021.

3. Abuhamad A, Minton KK, Benson CB, et al. Obstetric and gynecologic ultrasound curriculum and competency assessment in residency training programs: consensus report. J Ultrasound Med 2018;37(1):19-50. DOI: 10.1002/jum.14519

4. Green J, Kahan M, Wrong S. Obstetric and gynecologic resident ultrasound education. J Ultrasound Med 2015;34:1583-1589. DOI: 10.7863/ultra.15.14.10067. Epub 2015 Aug 7. PMID: 26254157.

5. Tolsgaard MG, Rasmussen MB Tappert C, et al. Which factors are associated with trainees' confidence in performing obstetric and gynecological ultrasound examinations? Ultrasound Obstet Gynecol 2014; 43(4):444-451. DOI: 10.1002/uog.13211

6. Moore CL, Copel JA. Point-of-care ultrasonography. N Engl J Med 2011;364(8):749-757. DOI: 10.1056/NEJMra1916062

7. Vallabh-Patel V, Mendez M, Kupesic Plavsic S. The importance of multimodality pelvic ultrasound simulation in teaching of obstetrics and gynecology residents. Donald School J Ultrasound Obstet Gynecol 2014;8(1):1-5. DOI: 10.5005/jp-journals-10009-1326

8. Madsen ME, Konge L, Nørgaard LN, et al. Assessment of performance measures and learning curves for use of a virtual-reality ultrasound simulator in transvaginal ultrasound examination. Ultrasound Obstet Gynecol 2014;44(6):693-699. DOI: 10.1002/uog.13400

9. Girzadas DV, Antonis MS, Zerth $\mathrm{H}$, et al. Hybrid simulation combining a high fidelity scenario with a pelvic ultrasound task trainer enhances the training and evaluation of endovaginal ultrasound skills. Acad Emerg Med 2009;16(5):429-435. DOI: 10.1111/j.1553-2712.2009.00399.x

10. Monsky WL, Levine D, Mehta TS, et al. Using a sonographic simulator to assess residents before overnight call. Am J Roentgenol 2002;178(1):35-39. DOI: 10.2214/ajr.178.1.1780035

11. Arya S, Diwedi A, Mulla Z, et al. Effectiveness of ultrasound simulation in obstetrics and gynecology education: a state-of-the-art review. Donald School J Ultrasound Obstet Gynecol 2017;11(2):115-125. DOI: 10.5005/jp-journals-10009-1512

12. Arya S, Mulla ZD, Kupesic Plavsic S. Role of pelvic ultrasound simulation. Clin Teach 2017;15(6):457-461. DOI: 10.1111/tct.12714

13. Alhramani L, Codsi E, Borowski KS. The current state of ultrasound training in obstetrics and gynecology residency programs. J Ultrasound Med 2018; 37(9):2201-2207. DOI: 10.1002/jum.14570

14. Benacerraf BR, Minton KK, Benson CB, et al. Proceedings: beyond Ultrasound First Forum on improving the quality of ultrasound imaging in obstetrics and gynecology. Am J Obstet Gynecol 2018; 218:19-28. DOI: 10.1016/j.ajog.2017.06.033

15. Lee W, Hodges AN, Williams $S$, et al. Fetal ultrasound training for obstetrics and gynecology residents. Obstet Gynecol 2004; 103(2):333-338. DOI: 10.1097/01.AOG.0000109522.51314.5c

16. Ahram J, Feinstein SJ, DePhillips H. Survey of ultrasound training in obstetrics and gynecology residency training programs in the United States. Obstet Gynecol 1987;70(3 pt 1):403-407.

17. American College of Obstetricians and Gynecologists. Practice Bulletin No 175: ultrasound in pregnancy. Obstet Gynecol 2016;128:e241-e256. DOI: 10.1097/AOG.0000000000001815

18. AIUM-ACR-ACOG-SMFM-SRU Practice parameter for the performance of standard diagnostic obstetric ultrasound examinations. J Ultrasound Med 2018;37(11):E13-E24. DOI: 10.1002/jum.14831

19. Calhoun BC, Hume RF. Integrated obstetric curriculum for obstetrics and gynecology residency, radiology residency and maternal-fetal medicine fellowship program at an accredited American Institute of Ultrasound in medicine diagnostic ultrasound center. Ultrasound Obstet Gynecol 2000;16(1):68-71. DOI: 10.1046/j.1469-0705.2000.00163.x

20. Council on Resident Education in Obstetrics and Gynecology. Educational objectives: core curriculum in obstetrics and gynecology. 2020. 12th edition. Available from: https://www.acog.org/educationand-events/creog/curriculum-resources/creog-educationalobjectives; Accessed May 29, 2021.

21. Bethune M, Aibrahim E, Davies B, et al. A pictorial guide for the second trimester ultrasound. Australas J Ultrasound Med 2013;16(3):98-113. 
DOI: 10.1002/j.2205-0140.2013.tb00106.x Available from: https:// www.aium.org/uls/index.htm; Accessed June 6, 2021.

22. Society for Maternal-Fetal Medicine, Stone JE, Kuller JA, Norton $M E$, et al. The Society for Maternal-Fetal Medicine (SMFM) fetal anomalies consult series. Am J Obstet Gynecol 2019;221(5):B2-B24. DOI: 10.1016/j.ajog.2019.08.014

23. Society for Maternal-Fetal Medicine, Benacerraf BR, Bromley B, Jelin, AC. SMFM fetal anomalies consult series \#1: facial anomalies. Am J Obstet Gynecol 2019;221(5):B3-B5. DOI: 10.1016/j.ajog.2019.08.048

24. Society for Maternal-Fetal Medicine, McKinney J, Rac MWF, Gandhi M. SMFM fetal anomalies consult series \#2: extremities.
Am J Obstet Gynecol 2019;221(6):B2-B18. DOI: 10.1016/j. ajog.2019.09.019

25. Society for Maternal-Fetal Medicine, Monteagudo A, Kuller J, Rotmensch S, et al. SMFM fetal anomalies consult series \#3: intracranial anomalies. Am J Obstet Gynecol 2020;223(6):B2-B50. DOI: 10.1016/j.ajog.2020.08.041

26. Farrell AS, Kuller JA, Boyd Bk, et al. Resident confidence with obstetrical ultrasound: are we meeting Council on Resident Education in Obstetrics and Gynecology objectives and Accreditation Council for Graduate Medical Education milestones? Am J Obstet Gynecol MFM 2020;2(4):100241. DOI: 10.1016/j.ajogmf.2020.100241 